2014. Demographics, symptoms at presentation and subsequent management were collected.

Results 132 records were returned with 89\% (117/131) identifying as men who have sex with men (MSM). 55\% (72/132) were HIV positive, with 3 new HIV diagnoses. 70\% (89/128) had symptoms of acute syphilis; with an ulcer, 87\% (41/47) had herpes simplex virus (HSV) PCR which was positive in $7 \%$ of cases. Dark ground microscopy was performed in 38\% (20/52) with one third being positive. $42 \%(56 / 131)$ were treated for syphilis on their initial visit (14\% if seen by a technician, $31 \%$ if seen by a nurse and $51 \%$ if seen by a medic, $\mathrm{p}=0.006) .90 \%$ had been treated by 2 weeks.

Discussion/conclusion MSM comprised the majority of acute syphilis with high rates of new HIV diagnoses, reinforcing the importance of routine HIV testing. There was a high co-infection prevalence of HSV. Dark ground microscopy was positive in a third of samples, perhaps due to technical difficulties in the clinic. Only 4 in 10 patients were treated at the first visit indicating a lack of awareness of symptoms of acute syphilis. More education on recognising and treating acute syphilis, especially in high risk groups, is needed.

\section{P165 SURVEY OF HEALTHCARE PROFESSIONALS' KNOWLEDGE AND ADHERENCE TO NATIONAL CHLAMYDIA SCREENING PROGRAMME GUIDANCE}

1,25usanna Currie*, ${ }^{2}$ Sarah C. Woodhall, ${ }^{2}$ Kevin Dunbar, ${ }^{2}$ John Saunders. ${ }^{1}$ Bolton NHS Foundation Trust, Bolton, Greater Manchester, UK; ${ }^{2}$ Public Health England, Colindale, London, UK

\subsection{6/sextrans-2016-052718.215}

Background The National Chlamydia Screening Programme (NCSP) aims to prevent and control chlamydia through detection and treatment of infection. The NCSP recommends that under 25 year-olds test annually, upon change of partner and retest three months after treatment. Healthcare professionals' (HCP) knowledge of and adherence to NCSP guidance is unknown.

Aims To establish HCPs' knowledge of and adherence to NCSP testing guidance, among those working in genitourinary medicine (GUM) and sexual and reproductive health (SRH) in England.

Methods Participants were invited through the BASHH newsletter and snowball sampling to complete an online survey (December 2015 to February 2016).

Results One hundred HCPs responded (82 medics, 17 nurses, 1 health adviser). Twelve percent knew the NCSP age limits (1524 years). Among respondents, 25\% identified screening criteria for annual testing, $70 \%$ for testing on change of partner, $59 \%$ for re-test following a positive and 16\% identified all three screening criteria. Of those who correctly identified screening criteria, $75 \%$ would always do it in practice, 19\% sometimes and $2 \%$ never. Of those who did not recognise screening criteria, $41 \%$ would still always screen appropriately in practice; 34\% sometimes; $10 \%$ never.

Discussion Knowledge of NCSP testing guidelines among healthcare providers was variable. While knowledge of NCSP was associated with testing in accordance with recommendations, knowledge did not automatically lead to adherence to testing recommendations. These findings will help to inform future development and dissemination of NCSP guidance.

\section{P166 SERVICE EVALUATION OF PERCEIVED NEEDS OF WOMEN LIVING WITH HIV IN THE OUTPATIENT SETTING}

${ }^{1}$ Rebecca Murdock*, ${ }^{1}$ Gladys Mundowa, ${ }^{1}$ Sonia Raffe, ${ }^{1,2}$ Daniel Richardson, ${ }^{1,2}$ Yvonne Gilleece. ${ }^{1}$ Brighton and Sussex University Hospitals, Brighton, UK; ${ }^{2}$ Brighton \& Sussex Medical School Brighton, UK

\subsection{6/sextrans-2016-052718.216}

Background/introduction Gender plays an important role in determining vulnerability and ability to access appropriate HIV care. Services must adapt to meet the needs of their population. Our HIV outpatient service provides care for 2400 people: $<15 \%$ are women

Methods A pilot plus follow up patient survey of the women attending the HIV outpatients.

Results 16 women completed the pilot questionnaire; 5/16 $(31.2 \%)$ aged $17-45$ years, $11 / 16(68.75 \%)$ aged $>46.4 / 16$ (25\%) disclosed a disability. 16/16 (100\%) had no difficulty accessing our service. 3/16 (18.8\%) of households had children living in them $<16$ years of age which $2 / 3(66 \%)$ attended with mother: $2 / 2 /(100 \%)$ were comfortable bringing their children into clinic. $1 / 3$ ( $33.4 \%$ ) had an option to leave children someone else. 10/16 (62.5\%) thought a service for women only would be useful: only $7 / 16$ (43.2\%) were aware of the nurse led Women only HIV service. Women found the following services most useful: counselling support/psychology 9/16 (56\%), cervical cytology 9/16 (56\%), menopausal advice 6/16 (37.5\%), benefitssupport $6 / 16(38 \%)$, sexual health screening $3 / 16(19 \%)$, fertility advice $3 / 16(18.8 \%)$, contraception advice $1 / 16(6 \%)$, and pregnancy advice 2/16 (13\%).8/16 (50\%) preferred a female HCP. 2/ $16(13 \%)$ reported violence or abuse from a partner or family member: $1 / 2(50 \%)$ of those discussed with a HCP.

Discussion/conclusion Preliminary results suggest that that the women attending our clinic have no issues with child care, language barriers or disabilities. Women over 45 years were more likely to take part in our study (70\% response). Of concern is a reported lack of knowledge about services already available which we are pursuing.

\section{P167 MANAGING RECTAL GC : ROOM FOR IMPROVEMENT}

Mannampallil Samuel, Amanda Samarawickrama, Arjun Sahota, Chris Taylor, Michael Brady*. King's College Hospital NHS Foundation Trust, London, UK

\subsection{6/sextrans-2016-052718.217}

Background/introduction Most ( 60\%) rectal gonorrhoea (GC) occur in MSM with $25 \%$ symptomatic at presentation. Those with rectal GC are at increased risk of other STIs. BASHH provide guidelines on GC management and targets to be achieved in testing, treatment and partner notification.

Aim(s)/objectives To compare our clinic's performance in managing rectal GC compared to the national recommendations.

Methods Retrospective case-note review of confirmed cases of rectal GC on NAAT between $1^{\text {st }}$ November 2011 and $31^{\text {st }}$ March 2015. Data were obtained from clinic notes, the clinic database and laboratory results. Audit standards were based on BASHH guidelines in managing GC.

Results 184 cases from 156 men: 61\% White, 12\% Black, median age 31 (IQR 26,37) years, 71\% MSM 29\% bisexual, $58 \%$ symptomatic. Triple site testing was done in $91 \%$. Rectal GC cultures were taken in $55 \%$. Adequate treatment was given to $94 \%$. Quinolone resistance occurred in 31\%. Partner notification was done in $43 \% .14 \%$ had other STIs (syphilis, LGV, 